\title{
O PROGRAMA FARMÁCIA POPULAR NO BRASIL: IMPORTÂNCIA, ABRAGÊNCIA E SEU IMPACTO NA SAÚDE
}

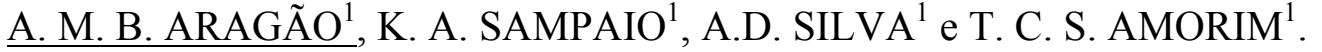 \\ ${ }^{1}$ Universidade Federal de Pernambuco, Centro de Ciências da Saúde, Departamento de \\ Ciências Farmacêuticas \\ E-mail para contato: adeny.aragao@gmail.com
}

\begin{abstract}
RESUMO - $O$ objetivo do estudo foi estabelecer uma relação entre o surgimento das farmácias de rede própria, que fazem parte do programa Farmácia Popular (PFP), e a ampliação do acesso aos medicamentos no País. Trata-se de um estudo retrospectivo, durante o período de 2004 à 2017. Os dados foram obtidos da SAGE (Sala de Apoio à Gestão Estratégica), instrumento que contém informações relacionadas ao SUS (Sistema Único de Saúde) e ao Ministério da Saúde. Foram coletados dados referentes ao número de farmácias populares no Brasil, nos estados e regiões. A análise estatística foi feita no programa Microsoft Excel ${ }^{\mathbb{B} .}$ A partir da análise dos dados verificou-se a existência de 497 farmácias populares da rede própria no Brasil, das quais 37,4\% se concentram na região Nordeste e 32,6\% na região Centro-Oeste. O estado que apresenta o maior número de farmácias foi São Paulo $(15,7 \%)$ e o estado com o menor número de farmácias foi Roraima (0,2\%). Observou-se que o número de farmácias da rede própria populares não é tão abrangente e que ainda é necessária uma maior ampliação do acesso aos medicamentos.
\end{abstract}

Palavras-chave: Saúde pública. Assistência farmacêutica. Planos e programas de saúde.

ABSTRACT - The objective of the study was to establish a relationship between the emergence of own network pharmacies, which are part of the Popular Pharmacy Program (PFP), and the expansion of access to medicines in the country. This is a retrospective study, during the 2004 to 2017. Data were obtained from the SAGE (Strategic Management Support Room), an instrument that contains information related to the SUS (Sistema Único de Saúde) and the Ministry of Health. Data were collected regarding the number of popular pharmacies in Brazil, in states and regions. Statistical analysis was performed in the Microsoft Excel ${ }^{\circledR}$ program. Based on data analysis, there were 497 popular pharmacies in Brazil's own network, of which 37.4\% are concentrated in the Northeast region and $32.6 \%$ in the Central-West region. The state with the highest number of pharmacies was São Paulo (15.7\%) and the state with the lowest number of pharmacies was Roraima (0.2\%). It has been observed that the number of popular pharmacies in the own network is not so comprehensive and that further expansion of access to medicines is still necessary.

Keywords: Public health. Pharmaceutical care. Health plans and programs. 


\section{INTRODUÇÃO}

O acesso à saúde é um direito do cidadão, garantido através da Constituição Federal de 1988. A lei orgânica da saúde 8.080/90, que regulamenta o Sistema Único de Saúde (SUS), estabelece os princípios de promoção, proteção e recuperação da saúde voltada para a população (COSTA; FRANCISCO; BARROS, 2014).

O Programa Farmácia Popular do Brasil foi criado em maio de 2004, com o objetivo de aumentar o acesso aos medicamentos essenciais e reduzir os gastos com medicamentos nos orçamentos familiares. Dessa forma, foi possível ampliar o acesso ao tratamento de diversas doenças, visto que muitas vezes os pacientes não completavam o tempo correto de tratamento devido a dificuldades financeiras que impediam a aquisição dos medicamentos. (BRASIL, 2005).

Apesar da criação de programas como a Política Nacional de Medicamentos (1998) e a Política Nacional de Assistência Farmacêutica (2004), o acesso e uso racional dos medicamentos ainda é um fator preocupante, pois não atinge a totalidade da população e em muitos locais ainda é possível verificar a indisponibilidade de medicamentos essenciais (BOING et al., 2013; VIEIRA, 2009).

Portanto, o objetivo do estudo é analisar a história do PFP no Brasil, realizando um levantamento das farmácias estatais existentes desde a criação do programa até o início de 2017 em nosso país. Além disso, avaliar a distribuição dessas farmácias nas regiões e estados do Brasil, estabelecendo uma relação entre o serviço prestado pelas farmácias de rede própria do programa e a ampliação do acesso aos medicamentos.

\section{MATERIAIS E MÉTODOS}

Os dados necessários a pesquisa foram obtidos através da SAGE (Sala de Apoio à Gestão Estratégica), um site pertencente ao governo que disponibiliza informações relacionadas aos indicadores de saúde e também contribui para a transparência das ações desenvolvidas na área de saúde. Logo após acessar o SAGE, clicando na opção "Saúde não tem preço - Assistência farmacêutica" e em seguida a opção "Farmácia popular do Brasil" é possível encontrar os dados que foram utilizados na pesquisa.

O estudo realizou um levantamento do número de farmácias do PFP no Brasil no período de 2004 a 2017, incluindo o número de farmácias no ano de 2017 de acordo com as regiões norte, nordeste, centro-oeste, sul e sudeste, das quais foram destacados os estados com maior e os com menor número de farmácias. O tratamento dos dados foi realizado através do programa Microsoft Excel $^{\circledR}$.

\section{RESULTADOS E DISCUSSÃO}

Ao longo dos anos o número de farmácias populares da vertente rede própria no Brasil aumentou de forma significativa. No período inicial do PFP o quantitativo de farmácias era menor, como pode ser observado na Tabela 1. Em 2004 havia apenas 27 destes estabelecimentos no país. A partir de 2006 é verificado um aumento expressivo desse número, no entanto em 2013 é evidenciado um declínio até 2017. 
Tabela 1 - Número de farmácias populares da rede própria em funcionamento no Brasil entre 2004 e início de 2017.

\begin{tabular}{|c|c|}
\hline ANO & FARMÁCIAS EM FUNCIONAMENTO \\
\hline 2004 & $\begin{array}{ll}27 & 27\end{array}$ \\
\hline 2005 & 75 \\
\hline 2006 & 259 \\
\hline 2007 & 407 \\
\hline 2008 & 504 \\
\hline 2009 & 530 \\
\hline 2010 & 543 \\
\hline 2011 & 555 \\
\hline 2012 & 558 \\
\hline 2013 & 546 \\
\hline 2014 & 532 \\
\hline 2015 & 523 \\
\hline 2016 & 517 \\
\hline 2017 & 497 \\
\hline
\end{tabular}

Fonte: Ministério da Saúde/ DAF/SCTIE/MS - Sala de Apoio à Gestão Estratégica, SAGE,2017.

Em relação às regiões do Brasil, no começo de 2017 as farmácias populares apresentam um percentual de $37,4 \%$ na região Nordeste, $15,1 \%$ na região Norte, $5,8 \%$ na região Centro-Oeste, $32,6 \%$ na região Sudeste e $9,1 \%$ na região Sul. É possível observar que as regiões Nordeste e Sudeste se sobressaem, corroborando com o estudo de Silva e Caetano (2015).

Dados referentes aos estados brasileiros revelaram que os três estados que apresentavam os maiores números de farmácias populares no início de 2017 eram: São Paulo (15,7\%), Bahia (11,5\%) e Pará (10\%). Os estados que apresentaram o menor número de farmácias foram: Roraima, possuindo apenas 1 farmácia, o que representa um percentual de $0,2 \%$ do total de farmácias populares no Brasil; logo em seguida Acre e Amapá, ambos com apenas 2 farmácias, representando um percentual de $0,4 \%$ cada.

O PFP é organizado em duas vertentes, a Rede Própria estatal e a "Aqui Tem Farmácia Popular" (ATFP), que funciona em parceria com o comércio farmacêutico (SILVA; CAETANO, 2015). Na rede própria são disponibilizados 112 itens, entre medicamentos e preservativo masculino, enquanto a ATFP possui uma lista diferente, atendendo a um público mais específico de pacientes.

Segundo o IBGE (2016), o Brasil apresenta 5.570 municípios. Destes munícipios, conforme o Ministério da Saúde (2017), 420 no início do ano de 2017 faziam parte da farmácia popular rede própria, número que se reduziu a apenas 10 municípios no final de 2017, sendo todas elas desabilitadas até o fim do ano. Em contrapartida, o número de farmácias da ATFP teve um crescimento muito significativo, possuindo mais de 30.000 unidades em 2016, segundo dados da SAGE. Portanto, os impactos desse acontecimento devem ser avaliados nos próximos anos, para que seja garantida a oferta de medicamentos do componente básico da assistência farmacêutica, assegurando a universalização do acesso à saúde.

\section{CONCLUSÃO}

A partir da análise das informações obtidas neste estudo foi possível observar que o número de farmácias populares da rede própria teve uma redução significativa e que ainda é necessária uma maior ampliação do acesso aos medicamentos. Apesar disso, a 
presença das farmácias do programa e a inserção de novas unidades para o incremento no atendimento às necessidades da população representou um avanço notável da assistência farmacêutica no país nos últimos, além de contribuir para a adesão aos tratamentos farmacológicos, aumentando a oferta de medicamentos a baixo custo.

\section{REFERÊNCIAS}

BOING, Alexandra Crispim et al. Acesso a medicamentos no setor público: análise de usuários do Sistema Único de Saúde no Brasil. Cad. Saúde Pública, Rio de Janeiro, v. 29, n. 4, p. 691-701, abr. 2013.

BRASIL. Ministério da Saúde. Fundação Oswaldo Cruz. Programa Farmácia Popular do Brasil: manual básico. Brasília: Ministério da Saúde, 2005.

BRASIL. Ministério da Saúde. Sala de Apoio a Gestão Estratégica. SUS. Número de municípios com farmácia popular- total Brasil. 2017.

COSTA, Karen Sarmento; FRANCISCO, Priscila Maria Stolses Bergamo; BARROS, Marilisa Berti de Azevedo. Conhecimento e utilização do Programa Farmácia Popular do Brasil: estudo de base populacional no município de Campinas-SP. Epidemiol. Serv. Saúde, Brasília, v. 23, n. 3, p. 397-408, set. 2014.

SILVA, Rondineli Mendes da; CAETANO, Rosangela. Programa "Farmácia Popular do Brasil": caracterização e evolução entre 2004-2012. Ciênc. saúde coletiva, Rio de Janeiro, v. 20, n. 10, p. 2943-2956, Out. 2015.

VIEIRA, Fabiola Sulpino. Gasto do Ministério da Saúde com medicamentos: tendência dos programas de 2002 a 2007. Rev. Saúde Pública, São Paulo, v. 43, n. 4, p. 674681, ago. 2009.

\section{AGRADECIMENTOS}

À Universidade Federal de Pernambuco (UFPE). 